\title{
Modelling Childbirth: Comparing Athlete and Non-athlete Pelvic Floor Mechanics
}

\author{
Xinshan $\mathrm{Li}^{1}$, Jennifer A. Kruger ${ }^{2}$, Jae-Hoon Chung ${ }^{1}$, Martyn P. Nash ${ }^{1}$, \\ and Poul M.F. Nielsen ${ }^{1}$ \\ ${ }^{1}$ Auckland Bioengineering Institute, The University of Auckland, New Zealand \\ shannon.li@auckland.ac.nz \\ ${ }^{2}$ Department of Sport and Exercise Science, The University of Auckland, \\ New Zealand
}

\begin{abstract}
There is preliminary evidence that athletes involved in highintensity sports for sustained periods have a higher probability of experiencing a prolonged second stage of labour compared to non-athletes. The mechanisms responsible for these differences are not clear, although it is postulated that muscle hypertrophy and increased muscle tone in athletes may contribute to difficulties in vaginal delivery. In order to test these hypotheses, we have constructed individual-specific finite element models of the female pelvic floor (one athlete and one non-athlete) and the fetal head to simulate vaginal delivery and enable quantitative analysis of the differences. The motion of the fetal head descending through the pelvic floor was modelled using finite deformation elasticity with contact mechanics. The force required to push the head was compared between the models and a $45 \%$ increase in peak force was observed in the athlete model compared to the non-athlete. In both cases, the overall maximum stretch was induced at the muscle insertions to the pubis. This is the beginning of a quantitative modelling framework that is intended to help clinicians assess the risk of natural versus caesarean birth by taking into account the possible mechanical response of pelvic floor muscles based on their size and activation patterns prior to labour.
\end{abstract}

\section{Introduction}

Recent clinical studies suggest that athletes who have engaged in long-term highintensity sports have a higher probability of experiencing a prolonged second stage of labour compared to non-athletes [1. Obstetricians and midwives have postulated that sustained training may lead to pelvic floor muscle hypertrophy, as well as changes in activation patterns, resulting in increased resistance during vaginal delivery.

One of the most important muscle groups involved in childbirth is the levator ani (LA). It consists of the iliococcygeus, pubococcygeus and puborectalis muscles. The latter two form a muscular sling around the anorectal junction, providing support for the urethra, vagina, and rectum. The iliococcygeus originates at the inner border of arcus tendinous and inserts posteriorly into the coccyx. On 
activation, these muscles lift upwards and pull the anorectal junction towards the back to narrow the genital hiatus [2].

For ethical and technical reasons, it is very difficult to record the LA muscle activity during childbirth. In order to gain a quantitative understanding of the role of pelvic floor muscles during childbirth, we have set up a modelling framework to simulate vaginal delivery using individual-specific pelvic floor geometries. In this study, we investigate the effect of muscle hypertrophy (in the absence of muscle activation) on the mechanical response of the pelvic floor by comparing models of an athlete and a non-athlete.

\section{Geometric Modelling}

\subsection{Female Pelvic Floor of Athlete and Non-athlete}

In a study conducted by Kruger et al 3, 27 nulliparous (women who have never given birth) underwent magnetic resonance (MR) imaging of the pelvic region, using a Siemens Magnetom Avanto 1.5T scanner. Two sets of MR images have been released to this study (due to ethical constraints): one from an athlete and one from a non-athlete. For each subject, thirteen components of the pelvic floor (see Fig. 1) were segmented and fitted using cubic Hermite finite element meshes to provide smooth $\mathrm{C}_{1}$-continuous geometric surfaces [4].
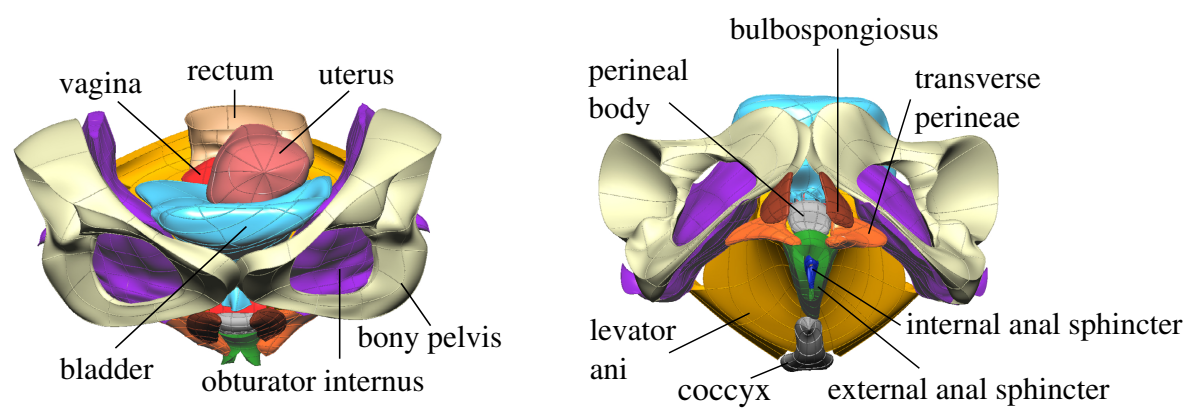

Fig. 1. The pelvic floor model (non-athlete) with thirteen components

Fig. 2 shows two MR slices proximal to the urethra for the athlete and the non-athlete. The LA muscles are outlined with dots. The difference in cross sectional area is clearly visible. Both of the LA meshes consist of 230 nodes and 88 tri-cubic Hermite hexahedral elements (5520 geometric degrees of freedom).

\subsection{Fetal Head}

The fetal head surface data was obtained by laser scanning a skull replica produced by ESP Ltd [5]. The region of the skull representing the vault (two frontal bones, two parietal bones, two temporal bones and one occipital bone) was used 

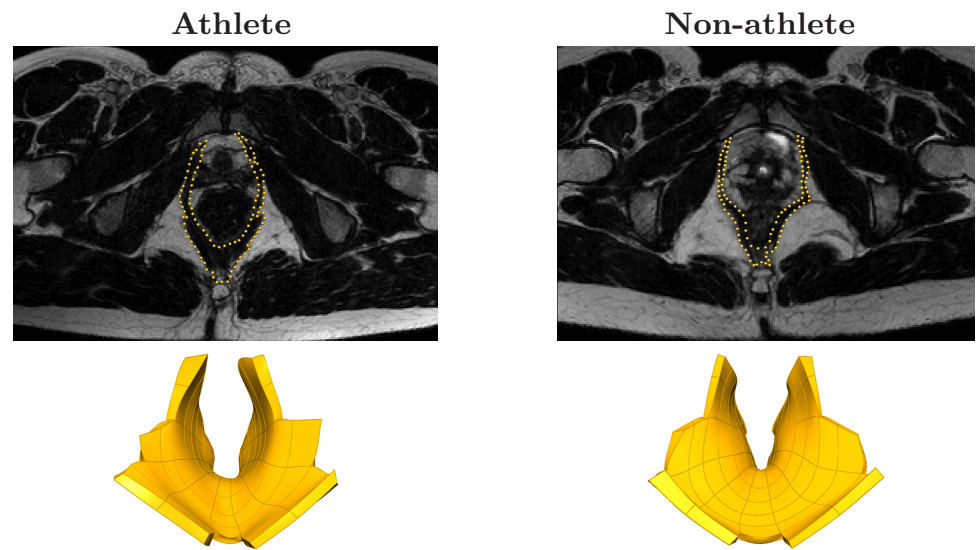

Fig. 2. MR images of the pelvic floor (above) and the tri-cubic Hermite meshes of the LA (below) for an athlete and a non-athlete

to create the fetal head model [2]. This area is in direct contact with the birth canal during the second stage of labour.

The given surface data was extrapolated into a volumetric data set using a constant $5 \mathrm{~mm}$ bone thickness. The fitted tri-cubic Hermite mesh consists of 280 nodes and 117 elements (6720 geometric degrees of freedom). Dimensions of the head are illustrated in Fig. 3 [2].

\section{Methods}

During the second stage of labour the head is pushed through the pelvis because of uterine and abdominal muscle (voluntary) contractions. The interactions between the head and the LA can be modelled using frictionless contact mechanics. Our in-house software CMISS1 was used to simulate this process. Sec. 3.1 provides a brief overview of the modelling framework. Please refer to Bonet and Wood [6], and Wriggers [7] for more details.

\subsection{Finite Deformation Elasticity Coupled with Contact Mechanics}

For finite deformation mechanics [6], the Lagrangian Green's strain tensor, E, is described as:

$$
\mathbf{E}=\frac{1}{2}\left(\mathbf{F}^{\mathbf{T}} \mathbf{F}-\mathbf{I}\right)=\frac{1}{2}(\mathbf{C}-\mathbf{I})
$$

$\mathbf{F}$ is the deformation gradient tensor, describing the mapping between two line segments $d \mathbf{X}$ and $d \mathbf{x}$, where $\mathbf{X}$ and $\mathbf{x}$ represent the initial and deformed

${ }^{1}$ An interactive computer program for Continuum Mechanics, Image analysis, Signal processing and System Identification, http://www.cmiss.org/ 

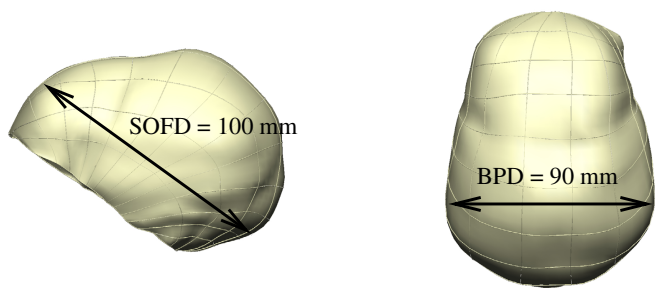

Fig. 3. Volumetric mesh of the fetal head in left lateral (left) and superior (right) views, $\mathrm{SOFD}=$ suboccipitofrontal diameter, $\mathrm{BPD}=$ biparietal diameter

configurations, respectively, $\mathbf{I}$ is the unitary tensor, and $\mathbf{C}$ is the right CauchyGreen deformation tensor. The square roots of the eigenvalues of $\mathbf{C}$ are the principal stretch ratios $\lambda_{\mathrm{i}}(\mathrm{i}=1 . .3)$.

The equations governing motion can be converted into a weak form using the principle of virtual work:

$$
\delta \mathrm{W}=\int_{V} \boldsymbol{\sigma}: \frac{\partial(\delta \mathbf{u})}{\partial \mathbf{x}} d V-\int_{V} \mathbf{b} \cdot \delta \mathbf{u} d V-\int_{S} \mathbf{t} \cdot \delta \mathbf{u} d S
$$

where $\boldsymbol{\sigma}$ is the Cauchy stress tensor, $\delta \mathbf{u}$ is the field of virtual displacements, $\mathbf{b}$ denotes the body force, and $\mathbf{t}$ is the surface traction. The integrals are performed over a reference volume $V$ and its bounding surface $S$. Here the principle of virtual work is modified to include two contact bodies and an extra contact term to penalise penetration between the two:

$$
\delta \mathrm{W}^{\text {coupled }}=\sum_{i=1}^{2} \delta \mathrm{W}^{(i)}-\int_{S_{c}^{(1)}} f_{c} \mathbf{n} \cdot\left[\delta \mathbf{u}^{(1)}-\delta \mathbf{u}^{(2)}\right] d S_{c}^{(1)}
$$

where $i$ is the index denoting the two contact bodies, $i=1$ is the slave (LA) and $i=2$ is the master (head), $\mathbf{n}$ is the normal vector defined at the master surface, $\delta \mathbf{u}^{(i)}$ are the virtual displacements associated with the slave or the master, and $S_{c}^{(1)}$ is the contact surface defined on the slave. The contact force $f_{c}$ is evaluated using the method of cross-constraints [8]:

$$
f_{c}=\left\{\begin{array}{lll}
\widehat{f}_{c} \cdot e^{\left(\widehat{k}_{c} / \widehat{f}_{c}\right) g} & g \leq 0 & \text { (no penetration) } \\
\widehat{f}_{c}+\widehat{k}_{c} g & g>0 & \text { (penetration) }
\end{array}\right.
$$

where $\widehat{f}_{c}$ and $\widehat{k}_{c}$ are estimates of the contact force and stiffness, respectively, and $g$ is the gap function, describing the amount of penetration.

\subsection{Modelling Parameters and Boundary Conditions}

Both the LA and the fetal head were assumed to be isotropic, homogeneous, and incompressible. Their mechanical behaviours were characterised using neo-Hookean material descriptions, where the strain energy function is described as:

$$
\Psi=c_{1}\left(I_{1}-3\right), \quad I_{1}=\operatorname{tr}(\mathbf{C})
$$


where $I_{1}$ is the first principal invariant of $\mathbf{C}$ and $c_{1}$ is a material constant. In this study, we have used a $c_{1}$ value of $10 \mathrm{kPa}$ for the LA muscle, which has been averaged from previous studies 910,11/2]. The $c_{1}$ value for the fetal head was assumed to be 10 times larger than that of the LA in order to differentiate the stiffness between fetal bone and maternal soft tissues.

Simulations were initiated by placing a well flexed head above the iliococcygeus. The head was then displaced through the LA over several steps until the biparietal diameter (BPD) was passed through the pelvis, which marked the crowning of the head. The degree of descent was controlled by altering the vertical coordinates for all top nodes of the head.

It was assumed that the head presents in an occiput anterior position (i.e., the fetus was facing the mother's back) on engaging the iliococcygeus and the muscle responded passively. By obstetrical conventions, the displacement of the fetal head was calculated with respect to a line joining the maternal ischial spines. A descent below the ischial spines was considered positive.

A support mesh was added to the LA, which represents arcus tendinous, part of obturator internus muscles, obturator fascia, and pubis at the front (see Fig. 1), all described by the same constitutive relations as the LA. The pelvic floor muscles were fixed at their attachment points to coccyx. The support mesh was fixed laterally at the ischial spines and anteriorly at the pubis.

\section{Results}

To quantify the level of difficulty during labour, we have estimated the reaction forces (by summing the vertical reactions at all top nodes) on the fetal head at each displacement step. These forces were normalised with respect to the maximum value found in the non-athlete model. Fig. 4 shows the evolution of normalised force versus fetal head descent. Simulations commenced at $25 \mathrm{~mm}$ and $10 \mathrm{~mm}$ below maternal ischial spines for the athlete and the non-athlete, respectively. This is caused by the difference in inter-individual anatomy, which leads to initial contacts at different positions. In both cases, the magnitude of the force exerted on the head increased monotonically until reaching a peak. The force then declined quickly and dropped below zero as the BPD descended out of the pelvis. A greater force was required to achieve delivery for the athlete compare to the non-athlete, with a $45 \%(1787 \mathrm{kN})$ increase in the peak value.

Fig. 5 illustrates the relationship between the principal stretch ratio and fetal head descent at four separate locations in the models. The overall maximum stretches were induced at positions $\mathrm{D}$ and $\mathrm{C}$ for the athlete and the non-athlete, respectively, and reached similar peak values (3.25 and 3.20, respectively). The stretch ratios at $\mathrm{A}$ and $\mathrm{B}$ were also similar.

\section{Discussion}

Biomechanical modelling of the second stage of labour is complicated because it requires detailed morphological descriptions of the pelvic floor structures, with 


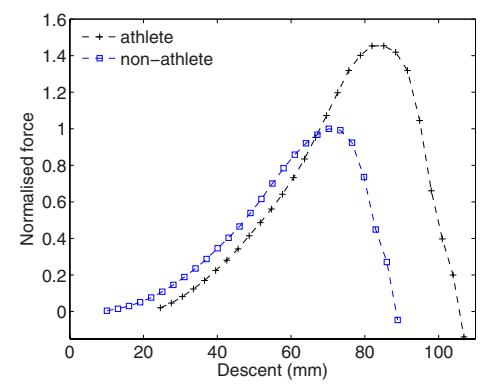

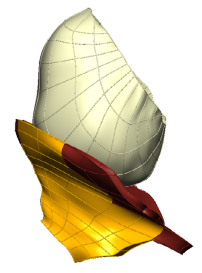

$25 \mathrm{~mm}$

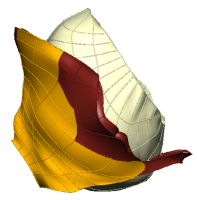

$85 \mathrm{~mm}$

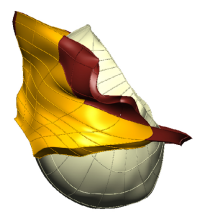

$107 \mathrm{~mm}$

Fig. 4. Normalised forces at each displacement step. The deformed models for athlete at $25 \mathrm{~mm}, 85 \mathrm{~mm}$, and $107 \mathrm{~mm}$ descent are shown in the right lateral view.

very sparse data 131415 . In this study, we have presented a quantitative modelling framework by integrating available information (e.g. detailed individualspecific pelvic floor geometries), to investigate regional loading and to compare pelvic floor muscle mechanics between an athlete and a non-athlete.

Our results indicate an increase in the peak force of $45 \%$ for the athlete, consistent with the need for more effort during delivery. The overall maximum principal stretches were induced at the lateral insertion points (to the pubis) for both models, with muscle elongations of more than 3.2 times their original length. However, in the case of the athlete, the maximum stretch was found in the support mesh instead of LA. As these results are limited by the assumptions made in the current model, further investigations are essential for other factors to be taken into account. For example, the pelvic floor muscles are unlikely to be passive without epidural anaesthesia during the second stage of labour. The difference in residual tone between athlete and non-athlete is likely to introduce significant changes in the stress distributions and thus kinematics. The effect of anisotropy will be studied in the future by introducing muscle fibers. Heterogeneity will also be incorporated into the model by defining different material parameters for tissues surrounding the LA. The material parameters are best determined experimentally, however few quantitative data are available. One possibility that we are investigating is the use of a compliance device to evaluate the pelvic muscle strength for each individual [16]. The birth process involves a series of movements of the fetal head (rotation, flexion and extension) in order to negotiate its way through the maternal pelvis, during which time the head 
Athlete
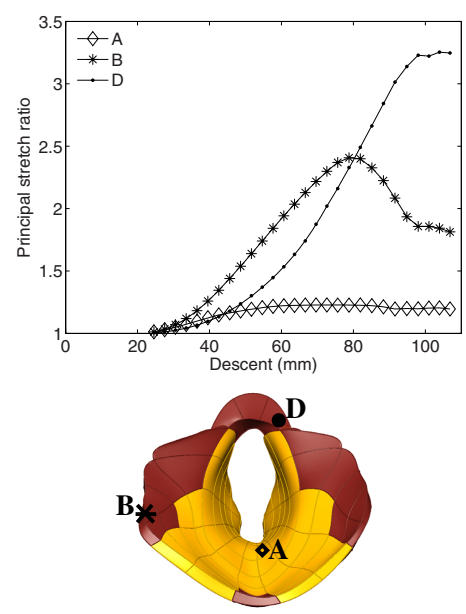

$25 \mathrm{~mm}$

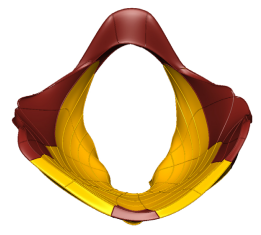

$85 \mathrm{~mm}$

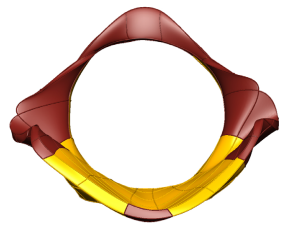

$107 \mathrm{~mm}$
Non-athlete
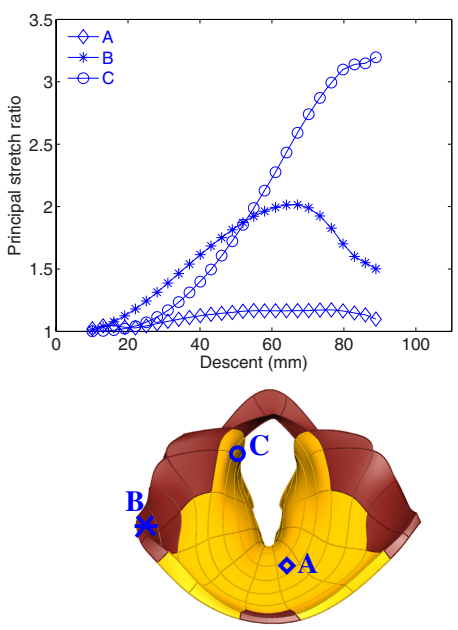

$10 \mathrm{~mm}$

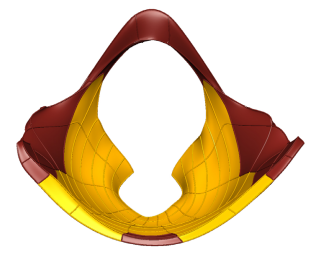

$70 \mathrm{~mm}$

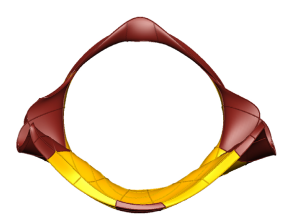

$89 \mathrm{~mm}$

Fig. 5. Principal stretch ratios versus fetal head descent at several locations in the pelvic floor muscles (above). Below are illustrations of the deformed pelvic floor muscles for athlete and non-athlete at specified descents.

moulds as part of the adaptation process 2. These changes, as well as the effects of the baby's body, will also need to be considered in the future.

\section{Acknowledgements}

We are thankful to Dr Rudy Lapeer at the University of East Anglia (UK) for providing the fetal head data via scanning the skull replica (produced by ESP Ltd). 


\section{References}

1. Kruger, J., Murphy, B., Thompson, S.: Childbirth and sportswomen: the perceptions of obstetric caregivers. Vision 14(2), 7-15 (2006)

2. Henderson, C., Macdonald, S.: Mayes' Midwifery: a Textbook for Midwives, 30th edn. Bailliere Tindall, Edinburgh (2004)

3. Kruger, J., Heap, S., Murphy, B., Dietz, H.: Pelvic floor function in nulliparous women using three-dimensional ultrasound and magnetic resonance imaging. Obstetrics \& Gynecology 111(3), 631-638 (2008)

4. Li, X., Kruger, J., Chung, J., Nash, M., Nielsen, P.: Modelling the pelvic floor for investigating difficulties during childbirth. In: Proceedings of SPIE 6916(69160V) (2008)

5. Lapeer, R., Prager, R.: Fetal head moulding: finite element analysis of a fetal skull subjected to uterine pressures during the first stage of labour. Journal of Biomechanics 34, 1125-1133 (2001)

6. Bonet, J., Wood, R.: Nonlinear Continuum Mechanics for Finite Element Analysis. Cambridge University Press, Cambridge (1997)

7. Wriggers, P.: Computational Contact Mechanics, 2nd edn. Springer, Heidelberg (2006)

8. Zavarise, G., Wriggers, P., Schrefler, B.: A method for solving contact problems. International Journal for Numerical Methods in Engineering 42(3), 473-498 (1998)

9. Herzog, W.: Skeletal Muscle Mechanics: From Mechanisms to Function. John Wiley \& Sons, Chichester (2000)

10. Lee, S., Darzi, A., Yang, G.: Subject specific finite element modelling of the levator ani. Medical Image Computing and Computer-Assisted Intervention 8, 360-367 (2005)

11. Ionescu, I., Guilkey, J., Berzins, M., Kirby, M., Weiss, J.: Computational simulation of penetrating trauma in biological soft tissues using the material point method. Studies in Health Technology and Informatics 111, 213-218 (2005)

12. Teran, J., Sifakis, E., Blemker, S., Hing, T., Lau, C., Fedkiw, R.: Creating and simulating skeletal muscle from the visible human data set. Institute of Electrical and Electronics Engineers Transactions on Visualization and Computer Graphics 11(3), 317-324 (2005)

13. Martins, J., Pato, M., Pires, E., Nataljorge, R., Parente, M., Mascarenhas, T.: Finite element studies of the deformation of the pelvic floor. Annals of the New York Academy of Sciences 1101(1), 316-334 (2007)

14. Hoyte, L., Damaser, M., Warfield, S., Chukkapalli, G., Majumdar, A., Choi, D., Trivedi, A., Krysl, P.: Quantity and distribution of levator ani stretch during simulated vaginal childbirth. Urogynecology (in press, 2008)

15. Lien, K., Mooney, B., DeLancey, J., Ashton-Miller, J.: Levator ani muscle stretch induced by simulated vaginal birth. The American College of Obstetricians and Gynecologists 103(1), 31-40 (2004)

16. Kruger, J., Murphy, B., Nielsen, P.: Pelvic floor muscle compliance in elite athletes. In: Abstract ICS Cairo 2008 (accepted, 2008) 